\title{
Exploiting Stochasticity for the Control of Transitions in Gyre Flows
}

\author{
Dhanushka Kularatne*, Eric Forgoston ${ }^{\dagger}$ and M. Ani Hsieh* \\ * Mechanical Engineering and Applied Mechanics, University of Pennsylvania, Philadelphia, PA, USA \\ Email: \{dkul, mya\}@seas.upenn.edu \\ ${ }^{\dagger}$ Department of Mathematical Sciences, Montclair State University Montclair, NJ, USA \\ Email: eric.forgoston@montclair.edu
}

\begin{abstract}
We present a control strategy to control the intergyre switching time of an agent operating in a gyre flow. The proposed control strategy exploits the stochasticity of the underlying environment to affect inter-gyre transitions. We show how control can be used to enhance or abate the mean escape time and present a strategy to achieve a desired mean escape time. We show that the proposed control strategy can achieve any desired escape time in an interval governed by the maximum available control. We demonstrate the effectiveness of the strategy in simulations.
\end{abstract}

\section{INTRODUCTION}

There is increased interest in the scientific community in employing autonomous marine vehicles (AMVs) to better understand various biological, chemical and physical processes in the ocean. Examples include characterizing the dynamics of plankton assemblages [1], measurement of temperature profiles [2], and monitoring of harmful algae blooms [3] and the dispersion of harmful contaminants [4]. These applications demand persistent monitoring of the relevant processes, and as such require the AMV-based sensors to operate for long durations of time. In addition, the sensors are often required to reside within a monitoring region of interest for specific amounts of time, and then move to adjacent regions to achieve more widespread sampling. However, the environment these sensors operate in is stochastic, nonlinear, and has high inertia, and the dynamics are not always well understood. Therefore, designing controllers for these sensors to reside in specific regions and/or to transition between regions, while minimizing actuation to prolong operation time, is extremely challenging.

The literature often utilizes simplified kinematic models for control of AMVs, ignoring the complex flow-vehicle dynamic interactions, which gives rise to modeling uncertainties. In this work, we present a method that exploits such modeling uncertainties and the inherent stochasticity of the environment, to control the dwell times of the sensors in a given region. We use a modeling framework where the ocean environment is considered to be composed of an array of gyre flow structures [5, 6], which can clearly be observed in satellite imagery of the ocean (see Figure 11. With the proposed control strategy, the average dwell time of a sensor in a gyre can be controlled to a desired value. Stochasticity in the environment induces transitions between adjacent gyres, which leads to reduced actuation energy requirements.

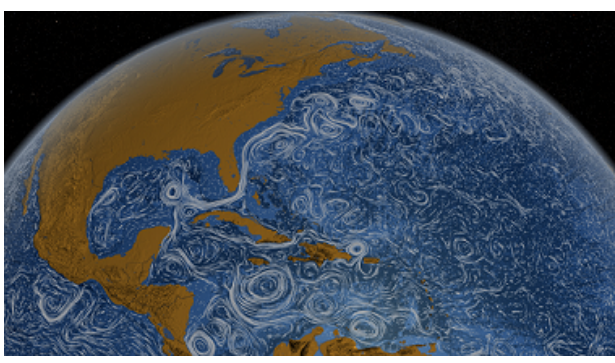

Fig. 1: Coherent structures, including gyres, in the ocean as observed by NASA satellites. Image from www.nasa.gov.

Recently, new mathematical tools have been developed to elucidate and harness the effects of noise on switching behavior in general dynamical systems [7, 8, 9]. These methods can predict the most probable switching path from one basin of attraction to an adjoining basin of attraction, that results from a large fluctuation due to the underlying noise in the system. More importantly, these methods can also accurately predict the expected switching time of particles between distinct basins of attraction. This framework has been used to describe a variety of physical and biological phenomena including extinction of diseases [10] or species [11] in populations, and switching between gene states [12] or magnetization states [13]. We use these techniques to predict the average time required to switch from one gyre to the next in an ocean environment. Since these transitions are precipitated by the noise in the system, they require little to no actuation energy. However, such noise-induced transitions are rare events, and as such cannot be relied upon for prolonged dwelling in a gyre or inter-gyre transition. Previous works [14, 15] have shown that the addition of limited controls can enhance or abate the switching times between gyres. However the control strategies proposed in those works cannot control the switching time to a desired value. In contrast, in the current work we present a strategy which uses limited control to achieve a desired average transition time. The required actuation is still minimal since the actual transition is affected by the noise in the system. The proposed strategy makes use of a few key assumptions in its derivation. Even with these assumptions, the problem of exploiting noise for navigation is a very complicated one, and to the best of our knowledge, this is the first attempt in the 


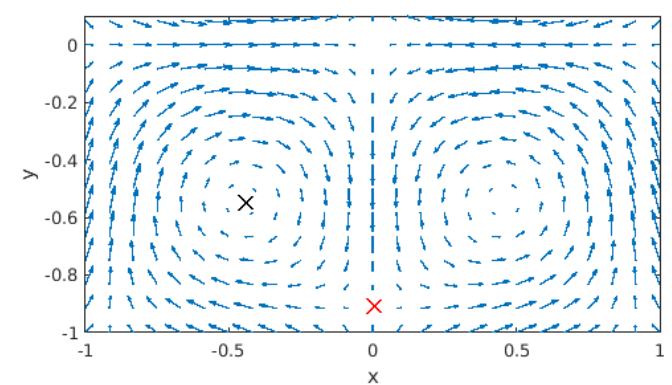

Fig. 2: Phase portrait of the double-gyre flow for $A=1, s=1$ and $\mu=1$. The black cross indicates the stable equilibrium at $\mathbf{x}_{\mathbf{0}}$, and the red cross indicates the saddle point at $\mathbf{x}_{\mathbf{1}}$.

literature at using control to obtain a desired mean escape time.

The rest of the paper is organized as follows: the problem is formulated in section II mean escape times are characterized in section IIII the proposed control strategy is presented in section IV, an analysis of the control is provided in section V], parameter selection for the control strategy is discussed in section VI, simulation results are presented in VII and conclusions and directions for future work are discussed in section VIII

\section{Problem Formulation}

We consider an agent with kinematics given by

$$
\dot{\mathbf{x}}=\mathbf{F}(\mathbf{x})+\mathbf{u}(t)+\boldsymbol{\eta}(t),
$$

where $\mathbf{x}=[x, y]^{T} \in \mathbb{R}^{2}$ is the position of the agent, $\mathbf{F}$ is the flow field in which the agent operates, $\mathbf{u}(t)$ is the agent control and $\boldsymbol{\eta}(t)$ is a white noise term where each component has zero mean and a standard deviation of $\sigma=\sqrt{2 D}$, with $D$ being the noise intensity. In this work, $\boldsymbol{\eta}(t)$ is used to capture any errors in modeling as well as any sensing, actuation, or environmental uncertainties. In ocean environments, a kinematic model is valid and sufficient to describe the dynamics when the vehicle dimensions are small in relation to the spatial scales of the transport controlling flow structures.

Here we assume the external flow field $\mathbf{F}(\mathbf{x})$ is given by the double-gyre model which is often used to describe large scale recirculation in the ocean [16]:

$$
\mathbf{F}(x, y)=\left[\begin{array}{l}
F_{x}(x, y) \\
F_{y}(x, y)
\end{array}\right]=\left[\begin{array}{c}
-\pi A \sin \left(\frac{\pi x}{s}\right) \cos \left(\frac{\pi y}{s}\right)-\mu x \\
\pi A \cos \left(\frac{\pi x}{s}\right) \sin \left(\frac{\pi y}{s}\right)-\mu y
\end{array}\right] .
$$

In (2), $A$ denotes the strength of the flow, $s$ is a scaling factor for the gyre dimensions, and $\mu$ is a damping coefficient. Figure 2 shows the phase portrait of the flow for $A=1, s=1$, and $\mu=1$. For $\mu>0$, each gyre has an attractor in the center of the gyre, and is flanked by four saddle points. The gyre boundaries consist of the stable and unstable manifolds of these saddle points. A system of two adjoining gyres as shown in Figure 2, qualitatively resembles a double-well potential.

To formalize the problem we consider a set of two adjoining gyres in the flow field. Let $\mathbf{x}_{0}$ be the attractor in the left gyre,

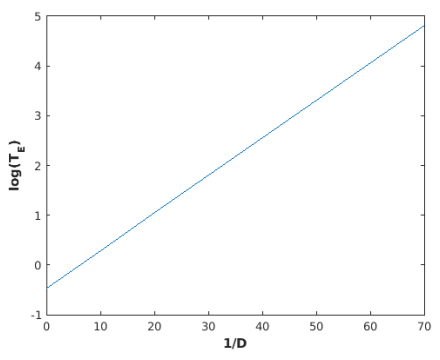

(a)

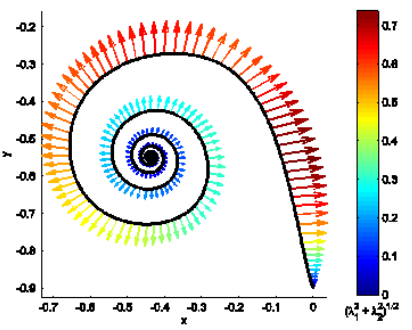

(b)
Fig. 3: (a) Average escape times obtained for the double gyre flow using Monte Carlo simulations. (b) The most likely escape path. Arrows along the escape path indicate the direction and strength of the most likely noise profile that leads to this most likely escape path.

and $\mathrm{x}_{1}$ be a saddle point at the lower right corner of the gyre. In the absence of noise and control, $\mathbf{x}_{0}$ is an attractor, and all passive agents in the left gyre will eventually converge to $\mathbf{x}_{0}$. In the presence of small noise, the dynamical behavior of the system is now determined by its stationary probability density. All the attractors at the gyre centers will now be peaks in this probability landscape, and most agent trajectories will now be concentrated around the gyre center. However, there exist rare events in which the noise in the system drives an agent from the attractor of one gyre to the attractor of an adjoining gyre. These "escape trajectories" most often pass through one of the saddle points since they are regions of high instability in the flow. The average time required for such an escape event to occur is called the mean escape time $T_{E}$, and it depends on the properties of the flow and the intensity of the noise in the system. The objective of the current work is to synthesize a control $\mathbf{u}(t)$ that achieves a desired escape time $T_{E}^{d}$. We assume that the agent has limited actuation and as such $\|\mathbf{u}(t)\| \leq c_{\max }$. We further assume that the agent is able to localize within each gyre, and that it is able to measure the flow velocity at its current position.

\section{Mean escape time due to noise}

In this section we characterize the mean escape time using large deviation theory. We first consider an uncontrolled system to obtain an expression for the mean escape time. Next we show how control could be used to increase or decrease the mean escape time.

\section{A. Mean escape times for the uncontrolled case}

From the theory of large deviations [17, 18], the probability of the uncontrolled system, i.e. $\mathbf{u} \equiv 0$, transitioning from the attractor at $\mathbf{x}_{0}$ through $\mathbf{x}_{1}$ to the adjoining gyre is given by

$$
P=k e^{-R / D}
$$

where $k$ is a constant and $R$ is the action of the transition path. Of the many paths leading to escape, the transition path that is most likely to occur will minimize the action. Using 
calculus of variations, one can show that the minimum action is given by,

$$
\begin{aligned}
\mathcal{R} & =\min _{\boldsymbol{\eta}(t)} \frac{1}{2} \int_{t_{0}}^{t_{f}} \boldsymbol{\eta}(t)^{T} \boldsymbol{\eta}(t) d t \\
& =\min _{\mathbf{x}(t)} \frac{1}{2} \int_{t_{0}}^{t_{f}}[\dot{\mathbf{x}}-\mathbf{F}(\mathbf{x})]^{T}[\dot{\mathbf{x}}-\mathbf{F}(\mathbf{x})] d t
\end{aligned}
$$

with boundary conditions $\mathbf{x}\left(t_{0}\right)=\mathbf{x}_{0}$, and $\mathbf{x}\left(t_{f}\right)=\mathbf{x}_{1}[19$ 17, 18, 20, 1 The average time to escape the gyre through $\mathbf{x}_{1}$ is inversely proportional to the probability of occurrence of this most likely transition path and its associated noise profile. Thus, the average escape time is given by,

$$
T_{E}=b e^{\mathcal{R} / D}
$$

where $b$ is a prefactor determined by numerical simulation or experiment. Figure $3 \mathrm{a}$ shows average escape times obtained through Monte-Carlo simulations for different noise intensities. It can be seen that the simulation results agree with the average escape time predicted by (5). Figure $3 \mathrm{~b}$ shows the escape path with the minimum action, i.e., the most likely escape path. The arrows along the path indicate the strength and direction of the noise profile associated with this most likely escape path. It can be seen that the noise acts as a control that pushes the agent out of the gyre.

In the limit of small noise, each escape trajectory is a rare event and thus the events are uncorrelated. Therefore, the escape events can be considered to be a Poisson process, and the probability density function of the escape times $P_{T_{E}}$ is exponential with a mean escape time of $T_{E}$, i.e.,

$$
P_{T_{E}}(t)=\frac{1}{T_{E}} e^{-\frac{t}{T_{E}}}, \quad t \geq 0
$$

\section{B. Mean escape time for the controlled case}

Inspired by the noise profile associated with the path most likely to occur, we consider a gyroscopic control force of the form $\mathbf{u}=c \mathbf{f}(\mathbf{x})$ to control the mean escape time. The function $\mathbf{f}(\mathbf{x})$ determines the direction of the control and it is assumed that $\|\mathbf{f}(\mathbf{x})\|=1$. Thus, $\|\mathbf{u}\|=c$. Similar to (4), the action of the path that is most likely to result in escape for this controlled system is given by,

$$
\mathcal{R}^{c}=\min _{\mathbf{x}(t)} \frac{1}{2} \int_{t_{0}}^{t_{f}}[\dot{\mathbf{x}}-\mathbf{F}(\mathbf{x})-c \mathbf{f}(\mathbf{x})]^{T}[\dot{\mathbf{x}}-\mathbf{F}(\mathbf{x})-c \mathbf{f}(\mathbf{x})] d t .
$$

Let the optimal escape path that results from (7) be denoted by $\mathbf{x}^{c}(t)$. Thus, the action of the most likely noise profile can be re-written as,

$\mathcal{R}^{c}=\frac{1}{2} \int_{t_{0}}^{t_{f}}\left[\dot{\mathbf{x}}^{c}-\mathbf{F}\left(\mathbf{x}^{c}\right)-c \mathbf{f}\left(\mathbf{x}^{\mathbf{c}}\right)\right]^{T}\left[\dot{\mathbf{x}}^{c}-\mathbf{F}\left(\mathbf{x}^{c}\right)-c \mathbf{f}\left(\mathbf{x}^{c}\right)\right] d t$.

When $c=0$, the action $\mathcal{R}^{0}$ is given by the uncontrolled case (4), and the corresponding escape path is $\mathrm{x}^{0}(t)$. Note that, for

\footnotetext{
${ }^{1}$ Due to the nature of the noise driven transition $t_{0}=-\infty$ and $t_{f}=\infty$.
}

an arbitrary $c$, the most likely path $\mathbf{x}^{c}(t)$ depends on $c$. Using a Taylor series expansion,

$$
\mathbf{x}^{c}(t)=\mathbf{x}^{0}(t)+\left.\frac{\partial \mathbf{x}^{c}}{\partial c}\right|_{c=0} c+\text { h.o.t. }
$$

Thus, for small values of $c$ such that the change in the optimal path is small, i.e., $\left|\frac{\partial \mathbf{x}^{c}}{\partial c}\right| \ll 1$, one has $\mathbf{x}^{c}(t) \approx \mathbf{x}^{0}(t)$. For small $c$, the action of the most likely escape path is therefore given by,

$$
\begin{aligned}
\mathcal{R}^{c} & \approx \frac{1}{2} \int_{t_{0}}^{t_{f}}\left[\dot{\mathbf{x}}^{0}-\mathbf{F}\left(\mathbf{x}^{0}\right)-c \mathbf{f}\left(\mathbf{x}^{\mathbf{0}}\right)\right]^{T}\left[\dot{\mathbf{x}}^{0}-\mathbf{F}\left(\mathbf{x}^{0}\right)-c \mathbf{f}\left(\mathbf{x}^{0}\right)\right] d t \\
& =\frac{1}{2} \int_{t_{0}}^{t_{f}}\left[\dot{\mathbf{x}}^{0}-\mathbf{F}\left(\mathbf{x}^{0}\right)\right]^{T}\left[\dot{\mathbf{x}}^{0}-\mathbf{F}\left(\mathbf{x}^{0}\right)\right] d t \\
& -c \int_{t_{0}}^{t_{f}} \mathbf{f}\left(\mathbf{x}^{\mathbf{0}}\right)^{T}\left[\dot{\mathbf{x}}^{0}-\mathbf{F}\left(\mathbf{x}^{0}\right)\right] d t+\frac{c^{2}}{2} \int_{t_{0}}^{t_{f}} \mathbf{f}\left(\mathbf{x}^{\mathbf{0}}\right)^{T} \mathbf{f}\left(\mathbf{x}^{\mathbf{0}}\right) d t .
\end{aligned}
$$

Concisely,

$$
\mathcal{R}^{c} \approx \mathcal{R}^{0}-\alpha c+\beta c^{2}
$$

where

$$
\alpha=\int_{t_{0}}^{t_{f}} \mathbf{f}\left(\mathbf{x}^{\mathbf{0}}\right)^{T} \boldsymbol{\eta}^{0}(t) d t, \quad \beta=\frac{1}{2} \int_{t_{0}}^{t_{f}} \mathbf{f}\left(\mathbf{x}^{\mathbf{0}}\right)^{T} \mathbf{f}\left(\mathbf{x}^{\mathbf{0}}\right) d t,
$$

and $\boldsymbol{\eta}^{0}(t)$ is the optimal noise profile for the uncontrolled case. Note that $\alpha>0$ and $\beta>0$. Thus, the change in action due to $c$ is given by

$$
\Delta \mathcal{R}=-\alpha c+\beta c^{2} .
$$

Using (5), the change in the escape time due to this change in the action is given by

$$
\frac{T_{E}^{c}}{T_{E}^{0}}=e^{\Delta \mathcal{R} / D}
$$

where $T_{E}^{0}$ is the mean escape time for the uncontrolled case. From (11) it can be seen that when $c<0$ then $\Delta \mathcal{R}>0$, which from (12) implies that $T_{E}^{c}>T_{E}^{0}$. Similarly, it can be seen that when $0<c<\alpha / \beta$ then $T_{E}^{c}<T_{E}^{0}$. Using (12), we can compute the value of $c$ required to affect a known change in the escape time.

\section{Controlling Escape Times in Gyre like flows}

Using (5), it can be seen that $\log \left(T_{E}\right)=\log (b)+\mathcal{R} / D$. Therefore, the average time required to escape from a gyre depends on the action as well as the amount of noise $D$ available in the system. For a given noise level in the system, the average escape time is governed by the action of the transition path that is most likely to occur.

The objective of this work is to use a control of the form $\mathbf{u}=c f(\mathbf{x})$, in which the parameter $c \in\left[-c_{\max }, c_{\max }\right]$ can be varied to obtain a given desired average escape time $T_{E}^{d}$. The function $f(\mathbf{x})$ determines the direction of the control vector, and is set as

$$
f(\mathbf{x})=\left[\begin{array}{lll}
0, & 0,1
\end{array}\right]^{T} \times \frac{\mathbf{F}(\mathbf{x})}{\|\mathbf{F}(\mathbf{x})\|},
$$

which results in a gyroscopic control vector that resembles the direction of the optimal noise profile [21, 5]. 


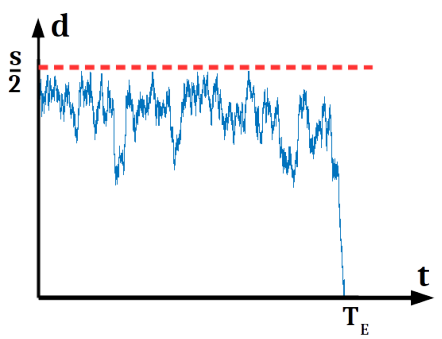

(a)

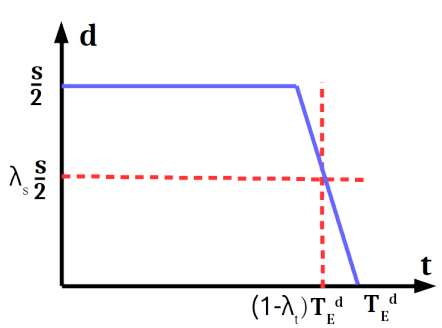

(b)
Fig. 4: (a) Variation of the distance to the closest gyre boundary over time along a noise-driven escape path. The width of the gyre is $s$. (b) The escape path hovers near the gyre center before exhibiting an almost linear transition towards escape.

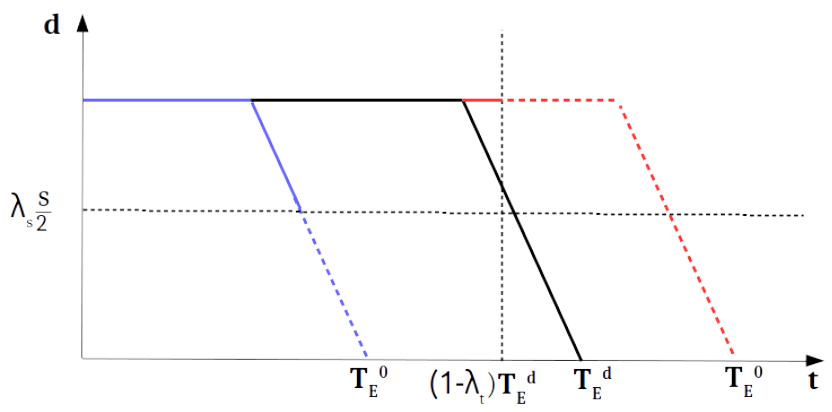

Fig. 5: Desired noise driven escape path with an escape time of $T_{E}^{d}$ (black); actual escape path when $T_{E}^{0}<T_{E}^{d}$ (blue); and actual escape path when $T_{E}^{0}>T_{E}^{d}$ (red).

If the noise intensity $D$ and the current average escape time $T_{E}^{0}$ were known, equations (12) and (11) could easily be used to compute the value of $c$ required to obtain the desired escape time. However, typically, none of these parameters are readily available in a real system. Thus, in order to design a control strategy to obtain the desired average escape time, we consider the characteristics of a noise-driven escape path of an agent in this double-gyre flow. Figure 4a shows the typical variation of the distance $d$ between the particle and the closest gyre boundary over time until the agent escapes the gyre through one of the boundaries. A simplified $t$ vs. $d$ plot that captures the essential characteristic of the curve is shown in Figure 4b. From these plots, it can be seen that a major portion of the particle's trajectory is concentrated around the gyre center, before the particle suddenly transitions out of the gyre. The actual transition itself occurs over a fraction of the overall dwell time, and near the transition, the $t$ vs. $d$ curve is approximately linear. These typical characteristics can be used to identify a potential onset of the escape portion of a trajectory, when neither the noise level of the system nor the expected escape times are known.

\section{A. Controller Synthesis}

Let $T_{E}^{0}$ be the 'natural' escape time of the uncontrolled system for an unknown noise level, and let $T_{E}^{d}$ be the desired average escape time. If the noise in the system is high, then $T_{E}^{0}<T_{E}^{d}$ (blue trace in Figure 5), and if the noise in the system is low, then $T_{E}^{0}>T_{E}^{d}$ (red trace in Figure 5). Note that $T_{E}^{0}$ of the system is unknown. Therefore, the proposed control strategy is based on making local assumptions about $T_{E}^{0}$, and is given by $\mathbf{u}=c f(\mathbf{x})$ where $c$ is given by:

$c= \begin{cases}\max \left\{\frac{\alpha-\sqrt{\alpha^{2}+4 \beta \Delta \mathcal{R}}}{2 \beta},-c_{\max }\right\} & \text { if } d<\lambda_{s} \frac{s}{2} \text { and } \\ & t<\left(1-\lambda_{t}\right) T_{E}^{d} \\ c_{\max } & \text { if } t \geq\left(1-\lambda_{t}\right) T_{E}^{d} \\ 0 & \text { otherwise }\end{cases}$

In other words, when $d<\lambda_{s} \frac{s}{2}$ and $t<\left(1-\lambda_{t}\right) T_{E}^{d}$ (e.g., dashed portion of the blue trace in Figure 5), the particle is assumed to be transitioning towards escape, and $T_{E}^{0}<T_{E}^{d}$. In this case, using the approximately linear escape transition behavior, $T_{E}^{0}$ is estimated to be,

$$
T_{E}^{0}=\frac{t}{1-\lambda_{t} \frac{2 d}{\lambda_{s} s}} .
$$

Using (12), the required change in action is computed to be

$$
\Delta \mathcal{R}=k \log \left(\frac{T_{E}^{d}}{T_{E}^{0}}\right),
$$

and using $(11)$, the control parameter is set as,

$$
c=\max \left(\frac{\alpha-\sqrt{\alpha^{2}+4 \beta \Delta \mathcal{R}}}{2 \beta},-c_{\max }\right) .
$$

When $t \geq\left(1-\lambda_{t}\right) T_{E}^{d}$ (e.g., dashed portion of the red trace in Figure 5, it is assumed that $T_{E}^{0} \geq T_{E}^{d}$, and that the particle has not started its transition towards escape. In contrast to the previous case, an estimate for $T_{E}^{0}$ cannot be obtained. Furthermore, in order to meet the expected escape time target, the particle must transition out as soon as possible. In this case, the control parameter is set as

$$
c=c_{\max } .
$$

The control strategy pushes the agent towards the center of the gyre if it gets close to the boundary before the required amount of time has elapsed, and it pushes the agent towards the boundary when the elapsed time is close to the required escape time. The instances at which the control is switched on are governed by the parameters $\lambda_{s}$ and $\lambda_{t}$. Note that $\lambda_{t} \leq 1$ and $0 \leq \lambda_{s} \leq 1$. Intuitively it can be seen that large values of $\lambda_{s}$ will increase the escape time and that large values of $\lambda_{t}$ will decrease the escape time. In order to analyze the proposed control strategy and verify its correctness, we map this twodimensional (2D) system to an analogous one-dimensional (1D) system, and analyze the corresponding 1D control strategy. This greatly simplifies the analysis while preserving the essential characteristics of the controlled system. Insights from the 1D system are then used to select values for $\lambda_{s}$ and $\lambda_{t}$. 


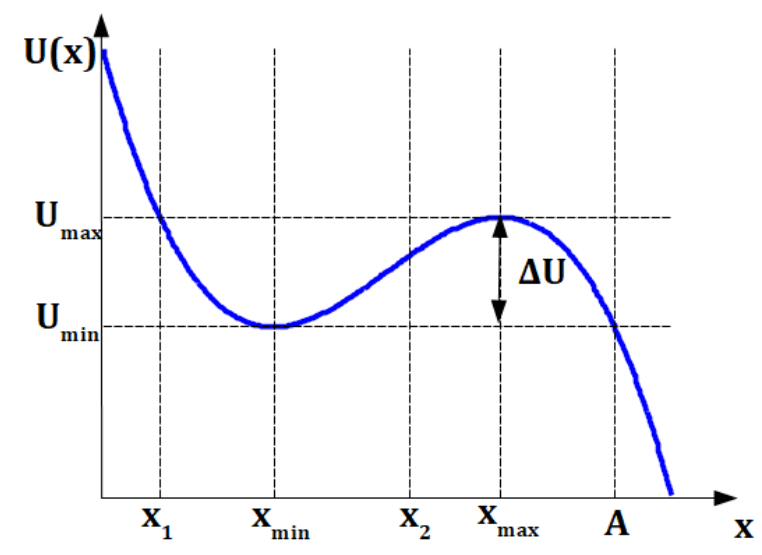

Fig. 6: Potential well $U(x)$.

\section{ANALYSIS OF THE CONTROL STRATEGY}

In order to analyze the proposed control strategy, we consider an analogous 1D system with an analogous control strategy. Consider a particle in a 1D potential well, subject to Gaussian noise. The equation of motion of this particle is given by

$$
\dot{x}=-\frac{\partial U}{\partial x}+\eta(t)+u(t),
$$

where $x$ is the position, $U$ represents the potential well (see Figure 6), $u(t)$ is the control, and $\eta$ is Gaussian noise with intensity $D$. For the uncontrolled case, i.e., $u(t)=0$, it has been shown [22, 23, 20] that if $\Delta U / D \gg 1$, the average time $\left(T_{E}\right)$ required for a particle to escape the stable equilibrium at $x_{\min }$ is given by

$$
\begin{aligned}
T_{E}^{0}= & \frac{1}{D} \int_{x_{1}}^{x_{2}} e^{-\left(\frac{U_{\min }}{D}+\frac{U_{\min }^{\prime \prime}}{2 D}\left(x-x_{\min }\right)^{2}\right)} d x \\
& \times \int_{x_{\min }}^{A} e^{\frac{U_{\max }}{D}-\frac{\left|U_{\max }^{\prime \prime}\right|}{2 D}\left(x-x_{\max }\right)^{2}} d x,
\end{aligned}
$$

where $U_{\min }^{\prime \prime}$ and $U_{\max }^{\prime \prime}$ are the second derivatives of $U(x)$ at $x_{\min }$ and $x_{\max }$ respectively, and $A$ is a point away from $x_{\max }$ as shown in Figure 6 Further details of this derivation can be found in a recent review [20]. Considering the exponential fall off of the integrands, the limits of both integrals can be extended from $-\infty$ to $\infty$, which gives,

$$
T_{E}^{0}=\frac{2 \pi}{\sqrt{U_{\min }^{\prime \prime}\left|U_{\max }^{\prime \prime}\right|}} e^{\frac{\Delta U}{D}} \text {. }
$$

This is the well known Kramers' escape rate for 1D systems [24].

Now, similar to the double-gyre flow, we consider a control action of $u(t)=c \frac{\partial U}{\partial x}$ with $c \leq c_{\max }<1$, and an analogous control strategy given by,

$$
c \begin{cases}<0, & x_{s} \leq x<x_{\max } \text { and } t<T_{t}, \\ >0, & t \geq T_{t} \\ =0, & \text { otherwise }\end{cases}
$$

where $x_{s}=x_{\max }-\lambda_{s}\left(x_{\max }-x_{\min }\right)$ and $T_{t}=\left(1-\lambda_{t}\right) T_{E}^{d}$, with $\lambda_{t} \leq 1$ and $0 \leq \lambda_{s} \leq 1$ The proposed control results in a controlled 1D system given by,

$$
\dot{x}=-(1-c) \frac{\partial U}{\partial x}+\eta(t) .
$$

This is equivalent to considering a potential well $\hat{U}=(1-c) U$. Thus, for $c<0$, the well becomes deeper and for $0<c<1$ the well becomes shallower. Substituting $U=\hat{U}$ in (17) and (18), we get,

$$
T_{E}^{c}=\frac{2 \pi}{(1-c) \sqrt{U_{\min }^{\prime \prime}\left|U_{\max }^{\prime \prime}\right|}} e^{(1-c) \frac{\Delta U}{D}}=\frac{e^{-c \frac{\Delta U}{D}}}{1-c} T_{E}^{0} .
$$

It can be shown that $c<0 \Rightarrow T_{E}^{c}>T_{E}^{0}$, and $0<c<1 \Rightarrow$ $T_{E}^{c}<T_{E}^{0}$ when $\Delta U / D \gg 1$, i.e., $c>0$ pushes the particle out towards the boundary and $c<0$ pulls the particle in towards the center of the well. Thus, this control is qualitatively similar to the gyroscopic control considered for the double-gyre flow.

Theorem 1. For a $1 D$ dynamical system given in (16) satisfying $\Delta U / D \gg 1$, in which the control is of the form $u(t)=c \frac{\partial U}{\partial x}$ where $c$ is selected according to (19), there exist $-c_{\max } \leq c \leq c_{\max }, \lambda_{t} \leq 1$ and $0 \leq \lambda_{s} \leq 1$ that can achieve any desired escape time $T_{E}^{d}$ satisfying

$$
T_{\min } \leq T_{E}^{d}<T_{\max }
$$

where

$$
T_{\min }=\frac{e^{-c_{\max } \frac{\Delta U}{D}}}{1-c_{\max }} T_{E}^{0}
$$

and

$$
T_{\max }=\frac{T_{E}^{0}}{4}\left(1+\frac{e^{-c_{\max } \frac{U_{\min }}{D}}}{\sqrt{1+c_{\max }}}\right)\left(1+\frac{e^{c_{\max } \frac{U_{\max }}{D}}}{\sqrt{1+c_{\max }}}\right) .
$$

Proof: To obtain an expression for the mean escape time under the proposed control strategy, we first consider applying a control with $c<0$ for $x_{s} \leq x<x_{\max }$, without considering the elapsed time. In this case, the control action can be written as $u(t)=-|c|\left(\Theta\left(x-x_{s}\right)-\Theta\left(x-x_{\max }\right)\right) \frac{\partial U}{\partial x}$, where $\Theta$ is a Heaviside function. Thus, the first integral $I_{1}$ of (17) for the mean escape time, can now be written as,

$$
\begin{aligned}
I_{1} & =\int_{x_{1}}^{x_{s}} e^{-\left(\frac{U_{\min }}{D}+\frac{U_{\min }^{\prime \prime}}{2 D}\left(x-x_{\min }\right)^{2}\right)} d x \\
& +\int_{x_{s}}^{x_{2}} e^{-(1+|c|)\left(\frac{U_{\min }}{D}+\frac{U_{\min }^{\prime \prime}}{2 D}\left(x-x_{\min }\right)^{2}\right)} d x .
\end{aligned}
$$

Considering that the integrands of both integrals decay exponentially, the lower limit of the first integral can be extended to $-\infty$, and the upper limit of the second integral can be extended to $\infty$. Thus,

$$
\begin{aligned}
I_{1} & =\sqrt{\frac{\pi D}{2 U_{\text {min }}^{\prime \prime}}} e^{-\frac{U_{\min }}{D}}\left(1+\operatorname{erf}\left(\sqrt{\frac{U_{\min }^{\prime \prime}}{2 D}}\left(x_{s}-x_{\text {min }}\right)\right)(23)\right. \\
& \left.+\frac{e^{-\frac{|c| U_{\min }}{D}}}{\sqrt{1+|c|}}\left(1-\operatorname{erf}\left(\sqrt{\frac{(1+|c|) U_{\min }^{\prime \prime}}{2 D}}\left(x_{s}-x_{\text {min }}\right)\right)\right)\right) .
\end{aligned}
$$


Using similar arguments, the second integral $I_{2}$ of (17), can be written as

$$
\begin{aligned}
I_{2}= & \sqrt{\frac{\pi D}{2\left|U_{\max }^{\prime \prime}\right|}} e^{\frac{U_{\max }}{D}}\left(2+\operatorname{erf}\left(\sqrt{\frac{U_{\max }^{\prime \prime}}{2 D}}\left(x_{s}-x_{\max }\right)\right)(24)\right. \\
& \left.-\frac{e^{\frac{|c| U_{\max }}{D}}}{\sqrt{1+|c|}} \operatorname{erf}\left(\sqrt{\frac{(1+|c|) U_{\max }^{\prime \prime}}{2 D}}\left(x_{s}-x_{\max }\right)\right)\right) .
\end{aligned}
$$

In (23) and 24], $\operatorname{erf}(x)=\frac{2}{\sqrt{\pi}} \int_{0}^{\infty} e^{-t^{2}} d t$. Thus, the expected escape time, when control of $c<0$ is enacted for $x_{s} \leq x<$ $x_{\max }$ is

$$
T_{E_{\text {dist }}}^{c}=\frac{1}{D} I_{1} I_{2}
$$

Next, we consider introducing a control with $c>0$ when $t \geq T_{t}$. Due to the stochastic nature of escape events, of the total paths that escape, $\int_{0}^{T_{t}} P_{T_{E}}(t) d t$ percent would have already escaped before the $c>0$ control is switched on at $t=T_{t} . P_{T_{E}}(t)=\frac{1}{T_{E_{d i s}}^{c}} e^{-\frac{t}{T_{E_{d i s t}}^{c}}}$ is the probability distribution of the escape times before switching on the $c>0$ control. Thus, the percentage of particles escaping after turning on the $c>0$ control is, $1-\int_{0}^{T_{t}} P_{T_{E}}(t) d t$, and the mean escape time for these particles would be $T_{E_{d i s t}}^{c}+T_{E}^{c}$, where $T_{E}^{c}$ is the mean escape time if the $c>0$ control is applied $\forall t \geq 0$, and is given in 21). Thus the expected mean escape time, under the full control strategy proposed in 19 is

$T_{E}^{e x p}=\int_{0}^{T_{t}} t P_{T_{E}}(t) d t+\left(T_{t}+T_{E_{d i s t}}^{c}\right)\left(1-\int_{0}^{T_{t}} P_{T_{E}}(t) d t\right)$.

Using (6), this can be simplified as

$$
T_{E}^{e x p}=T_{E_{d i s t}}^{c}-\left(T_{E_{d i s t}}^{c}-T_{E}^{c}\right) e^{-\frac{\left(1-\lambda_{t}\right) T_{E}^{d}}{T_{E}^{c}}}
$$

where $T_{E_{d i s t}}^{c}$ is given in 25, and $T_{E}^{c}$ is the nominal mean escape time with a control of $c>0$.

For $c=0$, it is trivial to see that $T_{E}^{c}=T_{E_{\text {dist }}}^{c}=T_{E}^{0}$. It can be shown that for $\Delta U / D \gg 1, \partial T_{E_{d i s t}}^{c} / \partial|c|>0$ and $\partial T_{E}^{c} / \partial c<0$. Thus, it can be inferred that, $T_{E_{d i s t}}^{c} \geq T_{E}^{c}$, with equality at the trivial case of $c=0$. Using this, one can easily show that $\frac{\partial T_{E}^{e x p}}{\partial \lambda_{t}}<0$ and is continuous for $\lambda_{t} \leq 1$. Thus, $T_{E}^{\exp }$ is minimized at $\lambda=1$ and it is maximized as $\lambda_{t} \rightarrow-\infty$. Thus, from (26), it can be seen that

$$
T_{E}^{c} \leq T_{E}^{e x p}<T_{E_{d i s t}}^{c},
$$

with $T_{E}^{e x p}=T_{E}^{c}$ for $\lambda_{t}=1$, and $T_{E}^{e x p} \rightarrow T_{E_{\text {dist }}}^{e}$ for $\lambda_{t} \rightarrow$ $-\infty$. Thus, for a given $c$ and $\lambda_{s}$, there exists $\lambda_{t} \leq 1$ that can achieve a desired escape time in the range established in 27].

As mentioned before, it can be shown that for $\Delta U / D \gg 1$, $\partial T_{E_{d i s t}}^{c} / \partial|c|>0$ and $\partial T_{E_{d i s t}}^{c} / \partial \lambda_{s} \mid>0$. In addition, $\left.T_{E_{\text {dist }}}^{c}\right|_{c=0}=T_{E}^{0}$ and $\left.T_{E_{\text {dist }}}^{c}\right|_{\lambda_{s}=0}=T_{E}^{0}$. Thus, the maximum of $T_{E_{d i s t}}$ occurs at $c=-c_{\max }$ and $\lambda_{s}=1$. Substituting these values in 25, we can get,

$$
T_{E}^{0} \leq T_{E_{\text {dist }}}^{c} \leq T_{\max }
$$

where

$$
T_{\max }=\frac{T_{E}^{0}}{4}\left(1+\frac{e^{-c_{\max } \frac{U_{\min }}{D}}}{\sqrt{1+c_{\max }}}\right)\left(1+\frac{e^{c_{\max } \frac{U_{\max }}{D}}}{\sqrt{1+c_{\max }}}\right) .
$$

Thus there exists a $\left(-c_{\max } \leq c \leq 0,0 \leq \lambda_{s} \leq 1\right)$ tuple that can achieve any $T_{E_{\text {dist }}}^{c}$ value in the range given in 28

In a similar fashion, one can show that there exists a $0 \leq$ $c \leq c_{\max }$ that can achieve any $T_{E}^{c}$ value in the range,

$$
T_{\min } \leq T_{E}^{c} \leq T_{E}^{0}
$$

where

$$
T_{\min }=\frac{e^{-c_{\max } \frac{\Delta U}{D}}}{1-c_{\max }} T_{E}^{0}
$$

From the above observations it can be concluded that there exist $-c_{\max } \leq c \leq c_{\max }, \lambda_{t} \leq 1$ and $0 \leq \lambda_{s} \leq 1$ that can achieve any desired escape time in the range $T_{\min } \leq T_{E}^{d}<$ $T_{\max }$.

Remark 1. In the above controller we consider $c<1$ in the analysis. If $c>1$, the peak and the trough of the effective potential $(1-c) U$ will be swapped, and the $\Delta U / D \gg 1$ assumption would not hold anymore.

\section{Vi. Controller Parameter Selection in 2D Gyre FLOWS}

If $T_{E}^{d}$ is inside the limits specified in Theorem 1 , there always exists a set of $\left(c, \lambda_{t}, \lambda_{s}\right)$ values that will achieve the desired escape time. If the noise intensity $D$ is known, depending on $T_{E}^{d}$, a suitable set of $\left(c, \lambda_{t}, \lambda_{s}\right)$ values can be selected to achieve $T_{E}^{d}$. In general, the noise level $D$ is not known. In such cases, not only is it impossible to determine a set of $\left(c, \lambda_{t}, \lambda_{s}\right)$ values to achieve a given $T_{E}^{d}$, but it is also not possible to determine if the required $T_{E}^{d}$ value is even feasible. In the analogous 2D gyre flow that we considered before, selecting a set of $\left(c, \lambda_{t}, \lambda_{s}\right)$ is even more complicated since an expression for $T_{E}$ of the form given in (21) is not available, and because the parameters of the flow are not known.

In the control strategy given in IV-A the problems outlined above are overcome by first selecting values for $c, \lambda_{t}, \lambda_{s}$ that approximately achieve the desired escape time for $T_{E}^{d}>T_{E}^{0}$, and then by refining $\lambda_{t}$ to achieve $T_{E}^{d}$ when $T_{E}^{d}<T_{E}^{0}$. For the $T_{E}^{d}>T_{E}^{0}$ case, we first approximately estimate the current uncontrolled escape time $T_{E}^{0}$ using (14), and then select a value for $c$ that would make $T_{E} \rightarrow T_{E}^{d}$ using $c<0$ control alone. Note that for this $T_{E}$ to be achieved using $c<0$ control alone, $\lambda_{s}=1$. Thus, for this case $T_{E_{d i s t}}^{c} \approx T_{E}^{d}$. According to $[26$, to make $T_{E}^{\exp } \approx T_{E_{\text {dist }}}^{c} \approx T_{E}^{d}$ we need $\lambda_{t} \rightarrow-\infty$. That is, by selecting a large value for $\lambda_{s}$ and a large negative value for $\lambda_{t}$, we are able to approximately achieve $T_{E}^{d}$ if $T_{E}^{d}>T_{E}^{0}$. However, if $T_{E}^{d}<T_{E}^{0}$, this large negative value for $\lambda_{t}$ will not be able to achieve the required $T_{E}^{d}$. Thus, in order to achieve the desired escape time for both $T_{E}^{d}>T_{E}^{0}$ and $T_{E}^{d}<T_{E}^{0}$, we select $0 \ll \lambda_{s}<1$ and $0<\lambda_{t} \ll 1$, i.e., $\lambda_{s}$ close to 1 , and $\lambda_{t}$ close to zero.

\section{RESULTS}

The control strategy given in section IV-A was used to control the escape time in a double-gyre flow field with parameters $A=1, \mu=1$ and $s=1$. The simulations considered a set of noise intensities $D=$ 
TABLE I: Values of $\lambda_{s}$ and $\lambda_{t}$ used in the simulations

\begin{tabular}{ccccccc}
\hline & set1 & set2 & set3 & set4 & set5 & set6 \\
\hline$\lambda_{s}$ & 0.85 & 0.85 & 0.85 & 0.2 & 0.2 & 0.2 \\
\hline$\lambda_{t}$ & -1 & 0.0625 & 0.9 & -1 & 0.0625 & 0.9 \\
\hline
\end{tabular}

$\{1 / 30,1 / 40,1 / 50,1 / 60,1 / 70\}$, and for each noise intensity, the following set of desired escape times $T_{E}^{d}=$ $\{3,6,12,26,57,122,262\}$ were considered. These $T_{E}^{d}$ values approximately correspond to the natural escape times $T_{E}^{0}$ for noise levels $\{1 / 20,1 / 30,1 / 40,1 / 50,1 / 60,1 / 70,1 / 80\}$ respectively. For each $\left(\mathrm{D}, T_{E}^{d}\right)$ pair, 1000 trials were simulated until escape from the gyre. In all simulations, $c_{\max }=0.5$ was considered. In order to investigate the effect of selecting different $\lambda_{s}$ and $\lambda_{t}$ values, simulations were run for the $\left(\lambda_{s}, \lambda_{t}\right)$ value combinations given in Table I In sets 1-3, a large value is selected for $\lambda_{s}(\approx 1)$, and in sets $4-6$, a small value is selected for $\lambda_{s}$. In both cases, $\lambda_{t}$ is successively increased from a negative value towards 1 . From the discussion in VI, the best results should be expected for set 2, where $\lambda_{s}$ is large and $\lambda_{t}$ is moderate. Figures $7 \mathrm{a} / 7 \mathrm{f}$ show plots of desired escape time $T_{E}^{d}$ vs the actual escape times $T_{E}^{a c t}$, for different $\left(\lambda_{s}, \lambda_{t}\right)$ value combinations. For each set of $\left(\lambda_{s}, \lambda_{t}\right)$ values, multiple noise levels are considered. In each figure, the thick dotted line in black represent the ideal $T_{E}^{d}=T_{E}^{a c t}$ curve. The closer the $T_{E}^{d} v s . T_{E}^{a c t}$ are to this line, the better the performance of the control strategy.

From the figures, it can be seen that set $2\left(\lambda_{s}=0.85, \lambda_{t}=\right.$ $0.0625)$ indeed gives the best results. In set $1, T_{E}^{a c t}$ overshoots $T_{E}^{d}$ by a considerable margin, since the negative value used for $\lambda_{t}$ cannot pull back $T_{E_{d i s t}}^{c}$ in 26) enough towards $T_{E}^{c}$. On the other hand, in set 3 , where $\lambda_{t}$ is close to $1, T_{E_{d i s t}}^{c}$ is pulled too far back by the $c>0$ control, which results in very small $T_{E}^{a c t}$ values. Set 4 and set 6 follow similar behaviors as set 1 and set 3 respectively due to the effect of $\lambda_{t}$. While sets 2 and 5 consider the same moderate value for $\lambda_{t}$, in set 5 , $T_{E}^{a c t}$ undershoots $T_{E}^{d}$ due to the small value of $\lambda_{s}$ considered in set 5 .

Figures $8 \mathrm{a} / \mathrm{e}$ show the proability densities of the escape times obtained for different values of $T_{E}^{d}$. For these simulations a noise level of $D=1 / 60$, which has a 'natural' escape time of approximately $57 \mathrm{~s}$, was considered. We used $\lambda_{s}=0.85$ and $\lambda_{t}=0.0625$ for the control. It can be seen that the proposed control is able to achieve $T_{E}^{d}$ values that are much farther away from the natural escape time. From these results, it can be seen that the control strategy proposed in section IV] is able to achieve a wide range of desired escape times, for a wide range of system noise levels.

The control strategy was also tested with a non-Gaussian noise source to check its performance in a non-ideal scenario. In this case the noise signal was derived as $\boldsymbol{\eta}(t)=\tilde{\sigma} \mathbf{z}^{1 / 3}(t)+\delta$ where each component of $\mathbf{z}$ has a standard normal distribution, i.e., $z_{i} \sim \mathcal{N}(0,1)$. The value for $\tilde{\sigma}$ was selected such that, the standard deviation of each component of $\boldsymbol{\eta}$ was equal to the standard deviations considered in the Gaussian case, i.e.,

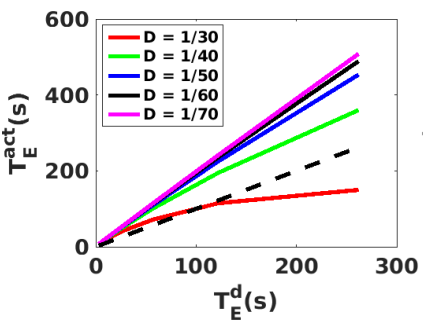

(a) set1

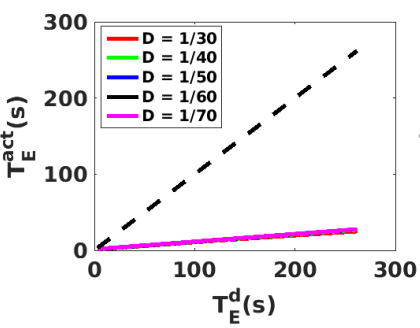

(c) set3

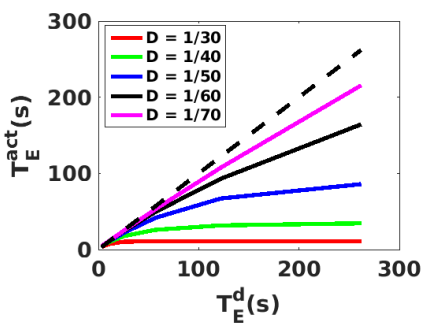

(e) set5

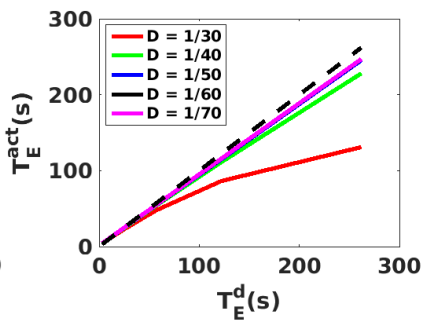

(b) set2

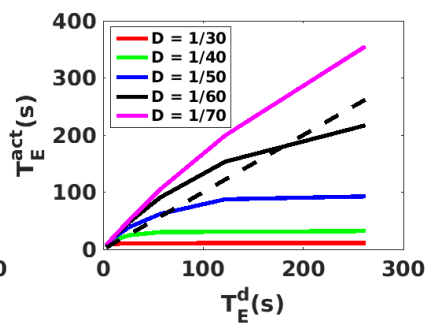

(d) set4

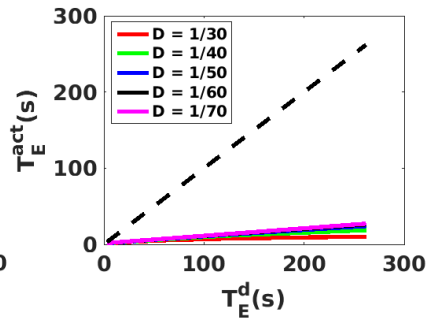

(f) set6

Fig. 7: Desired escape time $T_{E}^{d}$ vs. the actual escape times $T_{E}^{a c t}$ for different $\left(\lambda_{s}, \lambda_{t}\right)$ value combinations. The values of $\lambda_{s}$ and $\lambda_{t}$ for each set is given in Table $\mathrm{I}$

$\sigma\left(\eta_{i}\right)=\sqrt{2 D}$ for $i=1,2$. The mean $\delta$ essentially shifts the flow velocities in (2) by a constant amount, and its value is selected to be small enough such that the gyre structure of the flow is maintained. Figure 9 shows the results for the case where $\delta=0.1, \lambda_{s}=0.85$ and $\lambda_{t}=0.0625$. In the cases shown $\tilde{\sigma}$ was selected such that the noise signals have the same standard deviations as before. It can be seen that the desired mean escape times are achieved even in the presence of non-Gaussian noise sources.

\section{CONCLUSIONS}

In this work we presented a control strategy that could be used to control the inter-gyre switching time of an agent operating in a gyre flow. The proposed control strategy is derived from a framework based on large fluctuation theory which is used to characterize the mean escape time in uncontrolled dynamical systems. We showed how control can be used to enhance or abate the mean escape time and presented a strategy to achieve a desired mean escape time. We analyzed the control strategy using an equivalent 1D system, and showed that the proposed control strategy is able to achieve any desired escape time in an interval governed by 


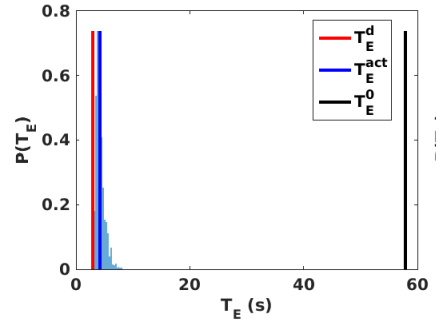

(a) $T_{E}^{d}=3 \mathrm{~s}$

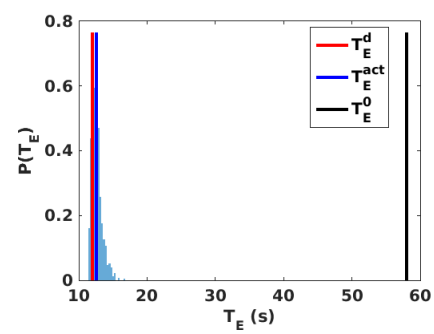

(c) $T_{E}^{d}=12 \mathrm{~s}$

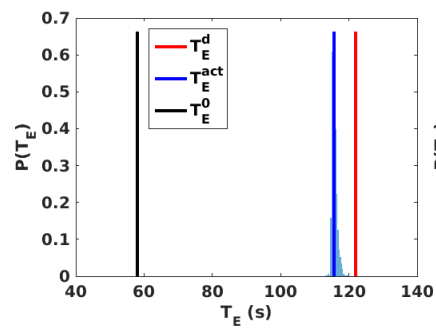

(e) $T_{E}^{d}=122$

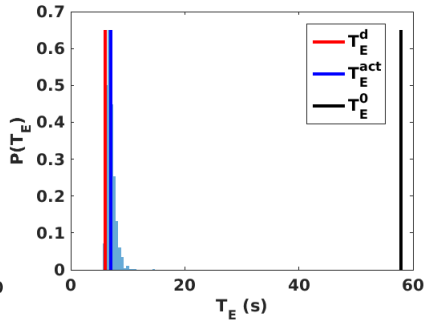

(b) $T_{E}^{d}=6 s$

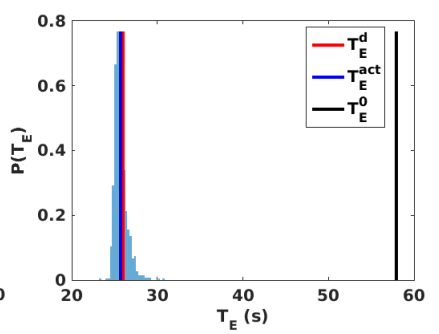

(d) $T_{E}^{d}=26 \mathrm{~s}$

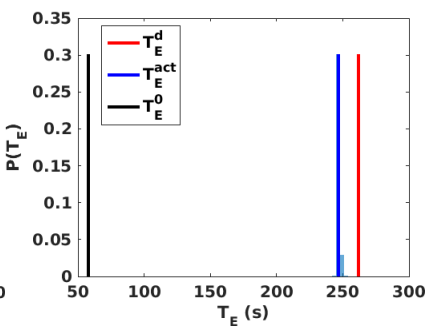

(f) $T_{E}^{d}=262 \mathrm{~s}$
Fig. 8: Probability density function of the actual escape times for various values $T_{E}^{d}$ for a noise level of $D=1 / 60$, where $\lambda_{s}=0.85$ and $\lambda_{t}=0.0625$ were used for the control.

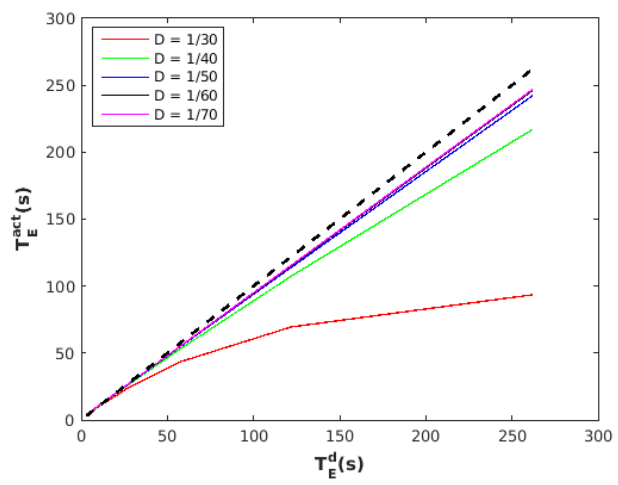

Fig. 9: $T_{E}^{d}$ vs. $T_{E}^{a c t}$ curves for non-Gaussian noise, where $\boldsymbol{\eta}(t)=\tilde{\sigma} \mathbf{z}^{1 / 3}(t)+\delta$ with $\delta=0.1, \lambda_{s}=0.85$ and $\lambda_{t}=0.0625$. $\tilde{\sigma}$ was selected such that $\sigma\left(\eta_{i}\right)=\sqrt{2 D}$ for $i=1,2$. the maximum available control. We showed how parameters of the control strategy can be picked in environments where full knowledge of the flow dynamics and stochasticity is not available. Finally, we demonstrated the effectiveness of the strategy in simulations, where we showed that the strategy works even in environments with non-Gaussian noise sources.

The presented method makes few key assumptions regarding the motion model and the noise model. While marine vehicles can be considered to be holonomic if the flow structures have a much larger spatial scale compared to the vehicle, they are non-holonomic in general. Furthermore, practical noise sources can be non-Gaussian and noise samples may be correlated in time. Even with these simplifying assumptions, obtaining a control law that achieves a given desired escape time is complicated. Relaxing these assumptions will sometimes require a new modeling paradigm and/or new theoretical results. Thus, evaluating the effects of modeling assumptions is a key area for future work. Towards this end we are currently designing and building an experimental setup to test the proposed methodology in a scaled gyre flow.

The presented methodology has potential applications in a wide range of disciplines. The method could potentially be used in other dynamical systems with multiple stability states to control switching between these states. For example, this framework could be used to study the noise-driven information state dynamics in a swarm using nonlinear consensus algorithms. Generalizing this method to work with such metastable dynamical systems is another direction for future work.

\section{ACKNOWLEDGEMENTS}

This work was supported by the Office of Naval Research (ONR) grant N000141712690 and the National Science Foundation (NSF) grants CMMI 1462825 and IIS 1253917.

\section{REFERENCES}

[1] D. Caron, B. Stauffer, S. Moorthi, A. Singh, M. Batalin, E. Graham, M. Hansen, W. Kaiser, J. Das, A. de Menezes Pereira, B. Z. A. Dhariwal, C. Oberg, and G. Sukhatme, "Macro- to fine-scale spatial and temporal distributions and dynamics of phytoplankton and their environmental driving forces in a small subalpine lake in southern California, USA," Journal of Limnology and Oceanography, vol. 53, no. 5, pp. 2333-2349, 2008.

[2] E. Fiorelli, N. E. Leonard, P. Bhatta, D. A. Paley, R. Bachmayer, and D. M. Fratantoni, "Multi-AUV Control and Adaptive Sampling in Monterey Bay," IEEE Journal of Oceanic Engineering, vol. 31, no. 4, pp. 935948, 2006.

[3] R. N. Smith, J. Das, H. Heidarsson, A. Pereira, I. Cetinić, L. Darjany, M.-E. Garneau, M. D. Howard, C. Oberg, M. Ragan, A. Schnetzer, E. Seubert, E. C. Smith, B. A. Stauffer, G. Toro-Farmer, D. A. Caron, B. H. Jones, and G. S. Sukhatme, "USC CINAPS builds bridges: Observing and monitoring the Southern California Bight," IEEE Robotics and Automation Magazine, Special Issue on 
Marine Robotics Systems, vol. 17, no. 1, pp. 20-30, 2010.

[4] H. Hajieghrary, D. Mox, and M. A. Hsieh, "Information theoretic source seeking strategies for multiagent plume tracking in turbulent fields," Journal of Marine Science and Engineering, vol. 5, no. 1, p. 3, 2017.

[5] C. R. Heckman, I. B. Schwartz, and M. A. Hsieh, "Toward efficient navigation in uncertain gyre-like flows," The International Journal of Robotics Research, vol. 34, no. 13, pp. 1590-1603, 2015.

[6] K. Mallory, M. A. Hsieh, E. Forgoston, and I. B. Schwartz, "Distributed allocation of mobile sensing swarms in gyre flows," Nonlin. Processes Geophys., vol. 20, no. 5, pp. 657-668, 2013.

[7] L. Billings, M. I. Dykman, and I. B. Schwartz, "Thermally activated switching in the presence of non-gaussian noise," Phys. Rev. E, vol. 78, p. 051122, 2008.

[8] E. Forgoston, L. Billings, P. Yecko, and I. B. Schwartz, "Set-based corral control in stochastic dynamical systems: Making almost invariant sets more invariant," Chaos, vol. 21, no. 1, p. 013116, 2011.

[9] H. B. Chan, M. I. Dykman, and C. Stambaugh, "Switching-path distribution in multidimensional systems," Phys. Rev. E, vol. 78, p. 051109, 2008.

[10] I. B. Schwartz, E. Forgoston, S. Bianco, and L. B. Shaw, "Converging towards the optimal path to extinction," Journal of The Royal Society Interface, vol. 8, no. 65, pp. 1699-1707, 2011.

[11] G. Nieddu, L. Billings, and E. Forgoston, "Analysis and control of pre-extinction dynamics in stochastic populations," Bulletin of mathematical biology, vol. 76, no. 12, pp. 3122-3137, 2014.

[12] M. Assaf, E. Roberts, and Z. Luthey-Schulten, "Determining the stability of genetic switches: Explicitly accounting for mRNA noise," Phys. Rev. Lett., vol. 106, p. 248102, 2011.

[13] R. V. Kohn, M. G. Reznikoff, and E. Vanden-Eijnden, "Magnetic elements at finite temperature and large deviation theory," Journal of nonlinear science, vol. 15, no. 4, pp. 223-253, 2005.

[14] C. R. Heckman, A. M. Hsieh, and I. B. Schwartz, "Going with the flow: Enhancing stochastic switching rates in multigyre systems," Journal of Dynamic Systems, Measurement, and Control, vol. 137, p. 031006, 2014.

[15] C. R. Heckman, M. A. Hsieh, and I. B. Schwartz, Experimental Robotics: The 14th International Symposium on Experimental Robotics. Springer International Publishing, 2016, ch. Controlling Basin Breakout for Robots Operating in Uncertain Flow Environments, pp. 561-576.

[16] G. Veronis, "Wind-driven ocean circulation Part 2. Numerical solutions of the non-linear problem," in Deep Sea Res. Ocean. Ab., vol. 13, no. 1. Elsevier, 1966, pp. 31-55.

[17] M. I. Freidlin and A. D. Wentzell, "On small random perturbations of dynamical system," Russian Math. Surveys, vol. 25 , pp. $1-55,1970$.

[18] — - Random Perturbations of Dynamical Systems. Springer-Verlag, 1984.

[19] R. P. Feynman and A. R. Hibbs, Quantum Mechanics and Path Integrals. McGraw-Hill, Inc., 1965.

[20] E. Forgoston and R. O. Moore, "A primer on noiseinduced transitions in applied dynamical systems," SIAM Review, 2017, accepted.

[21] C. R. Heckman, M. A. Hsieh, and I. B. Schwartz, "Controlling basin breakout for robots operating in uncertain flow environments," in International Symposium on Experimental Robotics (ISER 2014), Marrakech/Essaouira, Morocco, June 2014.

[22] N. G. Van Kampen, Stochastic processes in physics and chemistry. Elsevier, 1992, vol. 1.

[23] C. W. Gardiner, Handbook of stochastic methods for physics, chemistry and the natural sciences. SpringerVerlag, Berlin,, 2009.

[24] H. A. Kramers, "Brownian motion in a field of force and the diffusion model of chemical reactions," Physica, vol. 7, no. 4, pp. 284-304, 1940. 